Arq. Bras. Med. Vet. Zootec., v.56, n.2, p.258-263, 2004

\title{
Potencial forrageiro de híbridos de sorgo com capim-sudão
}

[Forage potential of sorghum sudangrass hybrids]

\author{
T.R. Tomich ${ }^{1}$, J.A.S. Rodrigues ${ }^{2}$, R.G.P. Tomich ${ }^{3}$, L.C. Gonçalves ${ }^{4}$, I. Borges ${ }^{4}$ \\ ${ }^{1}$ Embrapa Pantanal \\ Caixa Postal 109 \\ 79320-900 - Corumbá, MS \\ ${ }^{2}$ Embrapa Milho e Sorgo \\ ${ }^{3}$ Instituto de Ciências Biológicas da UFMG \\ ${ }^{4}$ Escola de Veterinária da UFMG
}

\section{RESUMO}

Avaliou-se o comportamento forrageiro de 23 genótipos experimentais e dois cultivares controle (AG2501C e BRS800) de híbridos de sorgo (Sorghum bicolor cv. Bicolor) com capim-sudão (Sorghum bicolor cv. Sudanense), utilizados em regime de corte. O delineamento experimental foi de blocos ao acaso com três repetições por tratamento e a comparação das médias foi feita pelo teste de Scott-Knott. A produção de matéria verde colhida 57 dias após o plantio variou de 24,8 a 37,8 t/ha, e a produção de matéria seca de 3,5 a $5,8 \mathrm{t} / \mathrm{ha}$, mas não houve diferença significativa entre híbridos. Houve grande variação quanto à relação folha/colmo, com diferença significativa entre os híbridos. A altura das plantas apresentou variações $(\mathrm{P}<0,05)$ entre híbridos e foi positivamente correlacionada $(\mathrm{P}<0,01)$ com as características de produção. A produtividade observada revela o potencial dos híbridos de sorgo com capim-sudão como alternativa para a produção de forragem. A diferença na relação colmo/folha pode servir como critério de seleção dos genótipos.

Palavras-chave: sorgo, capim-sudão, forragem cultivada, produtividade, regime de corte

\begin{abstract}
The objective of this study was to evaluated the forage potential of 23 experimental genotypes and two check cultivars (AG2501C and BRS800) obtained by crossing sorghum (Sorghum bicolor cv. Bicolor) with sudangrass (Sorghum bicolor cv. Sudanense), managed in a cutting regime. The experimental procedure followed a completely randomized block design, including three replications per treatment and the mean values were compared by Scott-Knott test. Fifty seven days after sowing, the fresh matter production varied from 24.8 up to $37.8 \mathrm{t} / \mathrm{ha}$, and dry matter production from 3.5 up to $5.8 \mathrm{t} / \mathrm{ha}$, both results were not statistically different. It was observed great variation to leaf/stem ratio, and difference $(P<0.05)$ among treatments. The height of the plants presented variation $(P<0.05)$ among hybrids and was positively correlated $(P<0.01)$ with the production characteristics. The productivity demonstrated the potential of these hybrids for forage production in central area of Brazil in the beginning of drought period. The significant difference in leaf/stem ratio could be used as a criterion for genotype selection.
\end{abstract}

Keywords: cultivated forage, cutting regime, productivity

Recebido para publicação em 23 de abril de 2003

Recebido para publicação, após modificações, em 18 de setembro de 2003

E-mail: thierry@cpap.embrapa.br 


\section{INTRODUÇÃO}

A restrição do consumo de nutrientes constitui, provavelmente, o principal fator capaz de limitar a produção de animais em pastejo, principalmente em regiões tropicais, onde ocorrem alterações significativas na composição e na disponibilidade das forrageiras ao longo do ano (t'Mannetje, 1983). O uso de forragem cultivada visa reduzir o efeito sazonal na produtividade e na qualidade das pastagens, aumentando a eficiência e a sustentabilidade produtiva e econômica da atividade pecuária.

Os híbridos de sorgo com capim-sudão apresentam rápido estabelecimento da cultura, alta velocidade de crescimento, boa capacidade de perfilhamento, resistência à seca, pouca exigência quanto à qualidade do solo e bom valor nutritivo como características desejáveis às plantas forrageiras (Bogdan, 1977; Wheeler, 1980). Esses híbridos têm sido utilizados no Sul do País como forragem cultivada de verão, com o objetivo de suprir as deficiências dos campos nativos da região (Zago, 1997). No Brasil Central, os híbridos de sorgo com capim-sudão, utilizados em corte ou pastejo, são adequados para atender à demanda por volumoso, que ocorre no final do outono, início do inverno, quando a disponibilidade de forragem no campo não é suficiente (Rodrigues, 2000), ou para garantir o suprimento de forragem no início do verão, período em que as pastagens ainda não se apresentam com o desenvolvimento satisfatório (Zago, 1997).

O potencial forrageiro de uma planta relaciona-se à sua capacidade produtiva e ao seu valor nutricional para a alimentação animal. Zago (1997) relatou produções significativamente superiores para dois híbridos de sorgo com capim-sudão, em relação às outras forrageiras de verão, testadas no Rio Grande do Sul. No Brasil Central, Rodrigues (2000) considera que alguns cultivares têm potencial para produzir até $90 \mathrm{t}$ de matéria verde por hectare, em três cortes. De acordo com Mulcahy et al. (1992), a utilização dos híbridos de sorgo com capim-sudão para a produção de forragem deve-se, entre outros fatores, à sua elevada produção de MS. Segundo os autores, os indicadores do valor nutritivo das forrageiras incluem as características físicas da planta. A determinação das correlações entre essas características e os parâmetros produtivos podem servir de base para a seleção de genótipos que apresentem alto valor forrageiro, ou seja, alto rendimento e valor nutricional. Embora tenha sido descrita por Zago (1997), a importância crescente do capim-sudão e de seus híbridos com o sorgo para a alimentação de rebanhos leiteiros e de corte em algumas regiões do País, a disponibilidade de cultivares para os produtores ainda é restrita. Este estudo foi desenvolvido com o objetivo de avaliar e comparar o potencial forrageiro, para utilização em regime de corte, de 23 genótipos experimentais e dois cultivares controle de híbridos de sorgo com capim-sudão.

\section{MATERIAL E MÉTODOS}

O experimento foi realizado em área da Embrapa Milho e Sorgo em Sete Lagoas, Minas Gerais. A região apresenta altitude média de 732 metros e clima do tipo AW (clima de savana, com inverno seco e temperatura média acima de $18^{\circ} \mathrm{C}$ no mês mais frio), segundo a classificação de Köppen. No dia 25 de outubro de 2002 foram plantados 23 genótipos experimentais: (A4*CMSXS169)*CMSXS912, (A3TX430*CMSXS225)*CMSXS912, (A3TX430*CMSXS227)*CMSXS912, (A3TX7000*CMSXS225)*CMSXS912, (A3TX7000*CMSXS227)*CMSXS912, (BR007*CMSXS217)*CMSXS912, (CMSXS210*CMSXS217)*CMSXS912, CMSXS210*CMSXS912, CMSXS211*CMSXS912, ATF54*CMSXS912, CMSXS157*CMSXS912,

CMSXS212*CMSXS912, CMSXS215*CMSXS912, ATF14*CMSXS912, ATF53*CMSXS912, CMSXS205*CMSXS912, CMSXS222*CMSXS912,

CMSXS156*CMSXS912, CMSXS206*CMSXS912, CMSXS218*CMSXS912, CMSXS220*CMSXS912, CMSXS107*CMSXS912, CMSXS216*CMSXS912 e dois cultivarescontrole (AG2501C e BRS800) de híbridos de sorgo com capim-sudão. Foram utilizados três canteiros (repetições) de $5 \mathrm{~m}$ de comprimento e quatro linhas, espaçadas em $0,70 \mathrm{~m}$ por tratamento. Foram feitas adubações no plantio, com $350 \mathrm{~kg} /$ ha da fórmula 04-14-08 (N:P:K), e em cobertura, $100 \mathrm{~kg} / \mathrm{ha}$ de uréia, de acordo com a análise do solo e as necessidades da cultura. 
Nas duas linhas centrais de cada canteiro, procedeu-se a contagem e a medição do número e da altura das plantas. O corte foi manual, cerca de $15 \mathrm{~cm}$ do solo, 57 dias após o plantio. Todo o material cortado foi pesado e o resultado utilizado para cálculo de produção de matéria verde. Em cada canteiro, seis plantas foram coletadas e separadas em partes, pesadas, présecadas em estufa de ventilação forçada, moídas em moinho com peneira de $1 \mathrm{~mm}$, amostradas e secas à $105^{\circ} \mathrm{C}$, até peso constante. Os resultados foram utilizados para a determinação da relação folha/colmo. O restante das plantas foi picado em partículas de aproximadamente $2 \mathrm{~cm}$, amostrado e pré-secado em estufa de ventilação forçada à $60^{\circ} \mathrm{C}$, por 48 horas. Após a pré-secagem, o material foi moído, amostrado e seco à $105^{\circ} \mathrm{C}$, até peso constante. Os resultados foram usados para a avaliação da produção de MS. Foi utilizado um delineamento de blocos ao acaso, com 25 híbridos e três repetições, sendo os blocos constituídos por três parcelas, cada uma contendo todos os híbridos. Para as análises utilizou-se o Sistema de Análises Estatísticas e Genéticas (Sistema..., 1983), segundo o modelo estatístico:

$\mathrm{Y}_{\mathrm{ij}}=\mu+\mathrm{B}_{\mathrm{i}}+\mathrm{H}_{\mathrm{j}}+\mathrm{e}_{\mathrm{ij}}$, em que:

$\mathrm{Y}_{\mathrm{ij}}=$ valor referente à observação do híbrido $\mathrm{j}$, no bloco i

$\mu=$ média geral

$\mathrm{B}_{\mathrm{i}}=$ efeito do bloco $\mathrm{i}(\mathrm{i}=1,2,3)$

$\mathrm{H}_{\mathrm{j}}=$ efeito do híbrido $\mathrm{j}(\mathrm{j}=1,2,3, \ldots, 25)$

$\mathrm{e}_{\mathrm{ij}}=$ erro aleatório associado à observação

Devido ao número elevado de tratamentos, para a comparação das médias foi empregado o teste de agrupamentos de Scott-Knott $(\mathrm{P}<0,05)$. Os coeficientes de correlação entre as variáveis foram estimados por meio do coeficiente de correlação de Pearson.

\section{RESULTADOS E DISCUSSÃO}

O número de plantas por hectare (Tab. 1), determinado no momento do corte, revelou diferença significativa entre os híbridos. Os genótipos

(A3TX430*CMSXS227)*CMSXS912, (A3TX7000*CMSXS225)*CMSXS912, (A3TX7000*CMSXS227)*CMSXS912, (CMSXS210*CMSXS217)*CMSXS912, ATF54*CMSXS912, CMSXS212*CMSXS912, CMSXS215*CMSXS912, ATF14*CMSXS912,
ATF53*CMSXS912, CMSXS220*CMSXS912, CMSXS216*CMSXS912 e os cultivarescontrole AG2501C e BRS800, com populações de 379,5 a 509,0 mil plantas/ha, foram superiores $(\mathrm{P}<0,05)$ aos demais. Os genótipos

(A4*CMSXS169)*CMSXS912, (A3TX430*CMSXS225)*CMSXS912, (BR007*CMSXS217)*CMSXS912, CMSXS210*CMSXS912, CMSXS211*CMSXS912, CMSXS157*CMSXS912, CMSXS205*CMSXS912, CMSXS222*CMSXS912, CMSXS156*CMSXS912, CMSXS206*CMSXS912, CMSXS218*CMSXS912 e CMSXS107*CMSXS912

apresentaram populações entre 287,1 e 359,0 mil plantas/ha. Como foram adotadas medidas necessárias para a uniformidade de plantio entre os tratamentos, possivelmente, a variação significativa no número de plantas por unidade de área está relacionada às diferenças na capacidade dos distintos híbridos para germinação, estabelecimento e perfilhamento nas condições do cultivo. Assim, o uso dos híbridos que apresentaram maior população de plantas pode determinar menor necessidade de tratos culturais para o desenvolvimento adequado da cultura.

A produção média de matéria verde variou de 24,4 a $37,8 \mathrm{t} / \mathrm{ha}$ e a de MS, de 3,5 a $5,8 \mathrm{t} / \mathrm{ha}$, contudo não houve diferença significativa entre os híbridos. A correlação entre a produção de matéria verde e a de MS foi de $0,96(\mathrm{P}<0,01)$. No Brasil, produtividade superior para híbridos de sorgo com capim-sudão, em manejo de corte, foi relatada por Zago (1997), enquanto que valores próximos ou rendimentos inferiores aos encontrados neste estudo foram citados por Rodrigues (2000) e por Tomich et al. (2001), respectivamente. Além da variabilidade genética, fertilidade do solo (Bogdan, 1977), disponibilidade de água (Rodrigues, 2000), época de plantio (Fribourg et al., 1975; Farias, Winch, 1987), estádio de desenvolvimento da planta (Worker Jr., Marble, 1968; Bishnoi et al., 1993), cortes sucessivos (Burger, Hittle, 1967) e número de plantas por unidade de área (Koller, Scholl, 1968; Worker Jr., 1973) são alguns dos fatores capazes de influenciar a produtividade de híbridos de sorgo com capim-sudão. Portanto, 
são irrelevantes as comparações entre estudos distintos.

Tabela 1. Número de plantas por hectare (Stand) e produções de matéria verde (MV) e de matéria seca (MS) de híbridos de sorgo com capim-sudão avaliados em regime de corte, plantados no mês de outubro em Sete Lagoas - MG, colhidos 57 dias após o plantio

\begin{tabular}{|c|c|c|c|}
\hline Híbrido & $\begin{array}{c}\text { Stand } \\
(1000 \text { plantas } / \mathrm{ha})^{\mathrm{b}}\end{array}$ & $\begin{array}{c}\text { Produção de MV } \\
(\mathrm{t} / \mathrm{ha})^{\mathrm{c}}\end{array}$ & $\begin{array}{c}\text { Produção de MS } \\
(\mathrm{t} / \mathrm{ha})^{\mathrm{d}}\end{array}$ \\
\hline$\overline{(\mathrm{A} 4 * \mathrm{CMSXS169)*CMSXS912}}$ & $358,6 \mathrm{~B}$ & 35,3 & 5,4 \\
\hline (A3TX430*CMSXS225)*CMSXS912 & $310,5 \mathrm{~B}$ & 24,4 & 3,7 \\
\hline (A3TX430*CMSXS227)*CMSXS912 & $398,1 \mathrm{~A}$ & 28,1 & 4,3 \\
\hline$(\mathrm{A} 3 \mathrm{TX} 7000 * \mathrm{CMSXS} 225) * \mathrm{CMSXS} 912$ & $405,7 \mathrm{~A}$ & 31,0 & 4,8 \\
\hline$(\mathrm{A} 3 \mathrm{TX} 7000 * \mathrm{CMSXS} 227) * \mathrm{CMSXS} 912$ & $385,2 \mathrm{~A}$ & 32,5 & 5,0 \\
\hline$(\mathrm{BR} 007 * \mathrm{CMSXS} 217) * \mathrm{CMSXS} 912$ & $347,1 \mathrm{~B}$ & 29,1 & 4,5 \\
\hline$(\mathrm{CMSXS} 210 * \mathrm{CMSXS} 217) * \mathrm{CMSXS} 912$ & $386,2 \mathrm{~A}$ & 27,6 & 4,2 \\
\hline CMSXS210*CMSXS912 & $324,7 \mathrm{~B}$ & 26,9 & 4,1 \\
\hline CMSXS211*CMSXS912 & $292,8 \mathrm{~B}$ & 36,3 & 5,3 \\
\hline ATF54*CMSXS912 & $422,4 \mathrm{~A}$ & 31,9 & 4,7 \\
\hline CMSXS157*CMSXS912 & $338,1 \mathrm{~B}$ & 24,5 & 3,5 \\
\hline CMSXS212*CMSXS912 & $407,1 \mathrm{~A}$ & 27,5 & 4,7 \\
\hline CMSXS215*CMSXS912 & $450,0 \mathrm{~A}$ & 25,6 & 4,1 \\
\hline ATF14*CMSXS912 & $450,9 \mathrm{~A}$ & 27,0 & 4,8 \\
\hline ATF53*CMSXS912 & $509,0 \mathrm{~A}$ & 35,2 & 5,0 \\
\hline CMSXS205*CMSXS 912 & $287,1 \mathrm{~B}$ & 29,4 & 4,1 \\
\hline CMSXS222*CMSXS 912 & $343,8 \mathrm{~B}$ & 27,6 & 4,4 \\
\hline CMSXS156*CMSXS912 & $359,0 \mathrm{~B}$ & 32,5 & 4,7 \\
\hline CMSXS206*CMSXS912 & $330,0 \mathrm{~B}$ & 31,0 & 4,7 \\
\hline CMSXS218*CMSXS912 & $349,5 \mathrm{~B}$ & 29,7 & 4,7 \\
\hline CMSXS220*CMSXS 912 & $379,5 \mathrm{~A}$ & 25,9 & 3,9 \\
\hline CMSXS107*CMSXS 912 & $306,2 \mathrm{~B}$ & 25,5 & 3,8 \\
\hline CMSXS216*CMSXS912 & $404,7 \mathrm{~A}$ & 25,0 & 3,9 \\
\hline AG2501C & $400,0 \mathrm{~A}$ & 37,8 & 5,8 \\
\hline BRS800 & $430,0 \mathrm{~A}$ & 27,0 & 4,0 \\
\hline Média & 375,0 & 29,4 & 4,5 \\
\hline
\end{tabular}

Médias seguidas por letras distintas na coluna diferem entre si pelo teste Scott-Knott $(\mathrm{P}<0,05) .{ }^{\mathrm{b}} \mathrm{CV}=13,8 \%,{ }^{\mathrm{c}} \mathrm{CV}=18,3 \%$, ${ }^{\mathrm{d}} \mathrm{CV}=20,0 \%$

A média de produção de matéria verde, 29,4 t/ha, aproxima-se do potencial de produção de diferentes híbridos, sob diversas condições de cultivo (Rodrigues, 2000). Levando-se em consideração que o plantio feito em outubro propicia cortes no período em que as pastagens do Brasil Central ainda estão se recuperando do período de estiagem, as produções médias encontradas podem ser consideradas satisfatórias. Também indicam que os híbridos de sorgo com capim-sudão avaliados podem ser uma alternativa para a produção de forragem cultivada no início do período chuvoso, isto é, ser uma opção para diminuir as necessidades de forragens conservadas e de concentrado nesse período.

As semelhanças estatísticas observadas para os dados de produtividade revelam que nenhum dos genótipos experimentais destacou-se em relação aos demais ou em relação aos cultivarescontrole. Dessa forma, estudos futuros com esses genótipos deverão compreender condições experimentais distintas ou, alternativamente, a seleção desses novos híbridos deverá abranger outras características, como o rendimento forrageiro em cortes sucessivos e/ou a produtividade em termos de MS digestível.

$\mathrm{Na}$ Tab. 2 estão as médias da relação folha/colmo e da altura das plantas. Foi notada diferença significativa entre os híbridos quanto à relação folha/colmo. $\mathrm{O}$ cultivar-controle BRS800 e os genótipos experimentais (A4*CMSXS169)*CMSXS912, (A3TX430*CMSXS225)*CMSXS912, CMSXS210*CMSXS912,

CMSXS211*CMSXS912 e ATF54*CMSXS912, com resultados entre 0,85 a 1,00 , apresentaram os maiores valores $(\mathrm{P}<0,05)$ de relação 
folha/colmo. Para o cultivar-controle AG2501C e demais genótipos experimentais essa relação variou de 0,57 a 0,81 . A relação folha/colmo é uma característica importante na previsão do valor nutritivo da forrageira. Ela indica a participação relativa de folhas na forragem total. Essa alta proporção constitui uma característica desejável para as plantas forrageiras, por estar diretamente associada à qualidade e ao consumo (Laredo, Minson, 1973). Dessa forma, o valor mais alto $(\mathrm{P}<0,05)$ da relação folha/colmo obtido para cinco dos 23 genótipos experimentais aponta no sentido de seleção desses genótipos, cuja forragem se antecipa ser de melhor qualidade.

Tabela 2. Relação folha/colmo e altura das plantas de híbridos de sorgo com capim-sudão avaliados em regime de corte, plantados no mês de outubro em Sete Lagoas - MG, colhidos aos 57 dias após o plantio

\begin{tabular}{|c|c|c|}
\hline Híbrido & Folha/Colmo ${ }^{b}$ & Altura $(\mathrm{m})^{\mathrm{c}}$ \\
\hline (A4*CMSXS169)*CMSXS912 & $1,00 \mathrm{~A}$ & $1,68 \mathrm{~A}$ \\
\hline$(\mathrm{A} 3 \mathrm{TX} 430 * \mathrm{CMSXS} 225) * \mathrm{CMSXS} 912$ & $0,84 \mathrm{~A}$ & $1,35 \mathrm{~B}$ \\
\hline$(\mathrm{A} 3 \mathrm{TX} 430 * \mathrm{CMSXS} 227) * \mathrm{CMSXS} 912$ & $0,72 \mathrm{~B}$ & $1,35 \mathrm{~B}$ \\
\hline$(\mathrm{A} 3 \mathrm{TX} 7000 * \mathrm{CMSXS} 225) * \mathrm{CMSXS} 912$ & $0,68 \mathrm{~B}$ & $1,50 \mathrm{~A}$ \\
\hline$(\mathrm{A} 3 \mathrm{TX} 7000 * \mathrm{CMSXS} 227) * \mathrm{CMSXS} 912$ & $0,67 \mathrm{~B}$ & $1,62 \mathrm{~A}$ \\
\hline$(\mathrm{BR} 007 * \mathrm{CMSXS} 217) * \mathrm{CMSXS} 912$ & $0,68 \mathrm{~B}$ & $1,48 \mathrm{~A}$ \\
\hline (CMSXS210*CMSXS217)*CMSXS 912 & $0,71 \mathrm{~B}$ & $1,58 \mathrm{~A}$ \\
\hline CMSXS210*CMSXS 912 & $0,95 \mathrm{~A}$ & $1,32 \mathrm{~B}$ \\
\hline CMSXS211*CMSXS912 & $1,00 \mathrm{~A}$ & $1,32 \mathrm{~B}$ \\
\hline ATF54*CMSXS912 & $0,98 \mathrm{~A}$ & $1,45 \mathrm{~B}$ \\
\hline CMSXS157*CMSXS912 & $0,76 \mathrm{~B}$ & $1,38 \mathrm{~B}$ \\
\hline CMSXS212*CMSXS 912 & $0,81 \mathrm{~B}$ & $1,57 \mathrm{~A}$ \\
\hline CMSXS215*CMSXS912 & $0,71 \mathrm{~B}$ & $1,40 \mathrm{~B}$ \\
\hline ATF14*CMSXS912 & $0,58 \mathrm{~B}$ & $1,62 \mathrm{~A}$ \\
\hline ATF53*CMSXS912 & $0,64 \mathrm{~B}$ & $1,53 \mathrm{~A}$ \\
\hline CMSXS205*CMSXS912 & $0,72 \mathrm{~B}$ & $1,37 \mathrm{~B}$ \\
\hline CMSXS222*CMSXS912 & $0,75 \mathrm{~B}$ & $1,52 \mathrm{~A}$ \\
\hline CMSXS156*CMSXS912 & $0,71 \mathrm{~B}$ & $1,52 \mathrm{~A}$ \\
\hline CMSXS206*CMSXS912 & $0,78 \mathrm{~B}$ & $1,27 \mathrm{~B}$ \\
\hline CMSXS218*CMSXS912 & $0,57 \mathrm{~B}$ & $1,70 \mathrm{~A}$ \\
\hline CMSXS220*CMSXS 912 & $0,80 \mathrm{~B}$ & $1,52 \mathrm{~A}$ \\
\hline CMSXS107*CMSXS912 & $0,74 \mathrm{~B}$ & $1,58 \mathrm{~A}$ \\
\hline CMSXS216*CMSXS912 & $0,77 \mathrm{~B}$ & $1,22 \mathrm{~B}$ \\
\hline $\mathrm{AG} 2501 \mathrm{C}$ & $0,60 \mathrm{~B}$ & $1,52 \mathrm{~A}$ \\
\hline BRS800 & $1,00 \mathrm{~A}$ & $1,42 \mathrm{~B}$ \\
\hline Média & 0,77 & 1,47 \\
\hline
\end{tabular}

Para a altura das plantas observou-se diferença significativa $(\mathrm{P}<0,05)$ entre os tratamentos. Treze genótipos experimentais e o cultivar-controle BRS800, com valores de 1,48 a $1,70 \mathrm{~m}$, foram superiores aos resultados obtidos para os demais híbridos, que variaram de 1,22 a $1,45 \mathrm{~m}$. A altura da planta é importante por ser uma característica normalmente correlacionada com as características de produção. Observaram-se correlações significativas entre a altura e as produções de matéria verde $(\mathrm{r}=0,47 ; \mathrm{P}<0,01)$ e de matéria seca $(\mathrm{r}=0,55 ; \mathrm{P}<0,01)$. Contudo, na seleção de genótipos para a produção de forragem, também é fundamental verificar as correlações existentes entre as características físicas indicadoras do valor nutritivo da forrageira. No presente estudo, a altura da planta apresentou correlação negativa $(\mathrm{r}=-0,33 ; \mathrm{P}<0,01)$ com a relação folha/colmo, observação geralmente indesejável quando se objetiva a produção de forragem de qualidade. Para híbridos de sorgo com capim-sudão, correlações negativas entre altura da planta e características de qualidade da forragem também foram verificadas por Mulcahy et al. (1992). Esses autores avaliaram sete híbridos e obtiveram correlações negativas entre altura e relação folha/colmo e altura e consumo. 


\section{CONCLUSÕES}

As produtividades médias de 29,4 t/ha de matéria verde e 4,5 t/ha de MS revelam o potencial dos híbridos de sorgo com capim-sudão estudados para serem utilizados como alternativa para a produção de forragem em regime de corte, no início do período chuvoso no Brasil Central. A relação folha/colmo pode ser usada para a seleção de híbridos experimentais. A correlação positiva entre altura da planta e rendimento forrageiro e negativa entre altura e relação folha/colmo indica a necessidade de cautela na utilização da altura como critério para seleção desses genótipos.

\section{REFERÊNCIAS BIBLIOGRÁFICAS}

BISHNOI, U.R.; OKA, G.M.; FEARON, A.L. Quantity and quality of forage and silage of pearl millet in comparison to Sudax, grain, and forage sorghuns harvested at different growth stages. Trop. Agric., v.70, p.98-102, 1993.

BOGDAN, A.V. Tropical pasture and fodder plants. New York: Longman, 1977. 475p.

BURGER, A.W.; HITTLE, C.N. Yield, protein, nitrate, and prussic acid content of sudangrass, sudangrass hybrids, and pearl millets harvested at two cutting frequencies and two stubble heights. Agron. J., v.59, p.259-262, 1967.

FARIAS, J.M.; WINCH, J.E. Effect of planting date and harvest stage upon yield, yield distribution and quality of sorghum sudangrass in northern Mexico. Trop. Agric., v.64, p.87-90, 1987.

FRIBOURG, H.A.; BRYAN, W.E.; BELL, F.F. et al. Performance of selected silage and summer annual grass crops as affected by soil type, planting date and moisture regime. Agron. J., v.67, p.643-647, 1975.

KOLLER, H.R.; SCHOLL, J.M. Effect of row spacing and seeding rate on forage production and chemical composition of two sorghum cultivars harvested at two cutting frequencies. Agron. J., v.60, p.456-459, 1968.
LAREDO, M.A.; MINSON, D.J. The voluntary intake, digestibility and retention time by sheep of leaf and stem fractions of five grasses. Aust. J. Agric. Res., v.24, p.875-888, 1973.

MULCAHY, C.; HEDGES, D.A.; RAPP, G.G. et al. Correlations among potential selection criteria for improving the feeding value of forage sorghums. Trop. Grassl., v.26, p.7-11, 1992.

RODRIGUES, J.A.S. Utilização de forragem fresca de sorgo (Sorghum bicolor x Sorghum sudanense) sob condições de corte e pastejo. In: SIMPÓSIO DE FORRAGICULTURA E PASTAGENS: TEMAS EM EVIDÊNCIA, 2000, Lavras. Anais... Lavras: UFLA, 2000. p.179-201.

SISTEMA de análises estatísticas e genéticas SAEG. Viçosa: UFV, 1983. 59p.

t'MANNETJE, J. Problem of animal production from tropical pastures. In: NUTRITION limits to animal production from pastures. Farnham Royal: CSIRO, 1983. p.67-85.

TOMICH, T.R.; RODRIGUES, J.A.S.; GONÇALVES, L.C. et al. Produção e proporções de folha e de colmo de doze híbridos de sorgo em manejo de corte, avaliados na rebrota. In: REUNIÃO ANUAL DA SOCIEDADE BRASILEIRA DE ZOOTECNIA, 38., Piracicaba, 2001. Anais... Piracicaba: SBZ, 2001. p. 291.

WHEELER, J.L. Increasing animal production from sorghum forage. World Anim. Rev., n.35, p.13-22, 1980.

WORKER Jr., G.F. Sudangrass and sorghum sudangrass hybrids responses to row spacing and plant maturity on yield and chemical composition. Agron. J., v.65, p.975-977, 1973.

WORKER Jr., G.F.; MARBLE, V.L. Comparison of growth stages of sorghum forage types as yield and chemical composition. Agron. J., v.60, p.669-672, 1968.

ZAGO, C.P. Utilização do sorgo na alimentação de ruminantes. In: MANEJO cultural do sorgo para forragem. Sete Lagoas: EMBRAPA/CNPMS, 1997. p.9-26 (Circular Técnica/EMBRAPA-CNPMS, 17). 